\title{
ESTUDO DA EXTRAÇÃO DE ÓLEO ESSENCIAL E DE COMPOSTOS BIOATIVOS DAS FOLHAS DE EUCALIPTO (Eucalyptus citriodora)
}

\author{
L. M. M. MACHADO ${ }^{1}$, R. S. NASCIMENTO ${ }^{1}$ e G. S. ROSA ${ }^{1}$ \\ ${ }^{1}$ Universidade Federal do Pampa, Curso de Engenharia Química \\ E-mail para contato: lauren_machado@hotmail.com
}

\begin{abstract}
RESUMO - O óleo essencial da espécie Eucalyptus citriodora é muito utilizado nas áreas de perfumaria, devido a seu aroma agradável. As folhas do eucalipto apresentam diversos compostos bioativos, sendo estes benéficos à saúde. O objetivo do presente trabalho foi estudar a eficiência de diferentes métodos de extração do óleo essencial presente nas folhas de eucalipto, além disso, determinar os compostos bioativos presentes nas folhas (clorofila e fenóis totais). Foram utilizadas amostras in natura e secas em estufa na temperatura de $60{ }^{\circ} \mathrm{C}$ por $2 \mathrm{~h}$. Para obtenção do óleo essencial empregaram-se duas metodologias: extrator Soxhlet e infusão. A determinação da clorofila foi realizada de acordo com o método de Lichtenthaler (1987), e a quantificação de fenóis por Singleton e Rossi (1965). Os resultados obtidos quanto às extrações do óleo essencial (em base seca) demonstraram que o emprego do Soxhlet, utilizando amostras previamente secas e como solvente o hexano, proporciona maior rendimento de óleo essencial. Observou-se que os conteúdos de fenóis e clorofila totais diminuíram para a amostra seca, quando comparado a amostra in natura, o que pode estar relacionado ao processo de secagem em estufa com temperatura de $60{ }^{\circ} \mathrm{C}$.
\end{abstract}

\section{INTRODUÇÃO}

O eucalipto é uma planta originária da Austrália, entretanto foi na China que se deu início a utilização do óleo essencial, tanto no tratamento estético da pele como em casos de queimaduras, feridas, bolhas, mordidas de insetos, piolhos e infecções cutâneas, em geral. O óleo presente nas folhas da espécie Eucalyptus citriodora vem sendo utilizado nas áreas de perfumaria, devido a seu aroma agradável, e farmacêutica, por apresentar diversos compostos bioativos benéficos à saúde. $\mathrm{O}$ eucalipto é indicado para o tratamento de constipações e gripes, sendo também eficiente contra dores musculares e em articulações. É um desintoxicante natural e revitaliza o sistema imunológico, respiratório e circulatório. Tem ainda seu espaço na aromaterapia, que indica o uso do eucalipto como estimulante mental, que melhora significativamente os níveis de concentração e de produtividade (Rocha e Santos, 2007). 
As folhas de eucalipto são tratadas como resíduo pela indústria da silvicultura (madeira, combustível, polpa de papel). Apesar de possuírem um baixo teor de óleos essenciais se comparadas às demais partes da árvore (como tronco e galhos), a grande quantidade de folhas disponíveis equilibra essa desvantagem. Para o Brasil, devido à grande área ocupada por reflorestamentos com eucalipto e à tendência para o aumento progressivo dessa cultura, os óleos essenciais de eucalipto se tornam um produto de crescente interesse econômico, contribuindo para a valorização da exploração florestal (Brito, 2002).

Estudos com diferentes espécies de eucaliptos mostraram que a extração alcança rendimentos de óleo essencial elevado quando ocorre a diminuição do conteúdo de umidade inicial por meio da secagem forçada de suas folhas (Braga et al., 2004). No que diz respeito a secagem de folhas, a secagem por leito fixo é muito pouco explorada. Na maioria das vezes, a secagem de folhas é feita de maneira natural, por exposição ao sol, ou mesmo a sombra, mesmo sendo um processo de difícil controle e dependente de condições climáticas. O processo de secagem de plantas aromáticas promove a conservação das propriedades físicas e químicas durante um maior período de tempo. No caso de plantas produtoras de óleo essencial, a secagem se torna mais criteriosa em razão da volatilidade dos óleos essenciais. Assim, a definição de metodologias mais apropriadas é necessária, visando assegurar os teores de substâncias ativas (Corrêa et al., 2004).

O presente trabalho teve como objetivo estudar a eficiência de diferentes métodos de extração do óleo essencial presente nas folhas de eucalipto (Eucalyptus citriodora), e além disso, determinar os compostos bioativos presentes nas folhas (clorofila e fenóis totais).

\section{MATERIAIS E MÉTODOS}

\subsection{Matéria-prima}

As amostras de folhas de eucalipto, após coletadas, foram armazenadas sob refrigeração e ausência de luminosidade. Parte da amostra foi seca em estufa a $60{ }^{\circ} \mathrm{C}$ por $2 \mathrm{~h}$. $\mathrm{O}$ conteúdo de umidade das folhas in natura e secas foi determinado pelo método da $\mathrm{AOAC}$ em estufa a $105^{\circ} \mathrm{C}$ por $24 \mathrm{~h}$.

\subsection{Procedimento experimental e Equipamentos}

As amostras utilizadas encontram-se na Figura 1, sendo folhas in natura (a) e folhas secas em estufa por $2 \mathrm{~h}$ a $60{ }^{\circ} \mathrm{C}(\mathrm{b})$. 


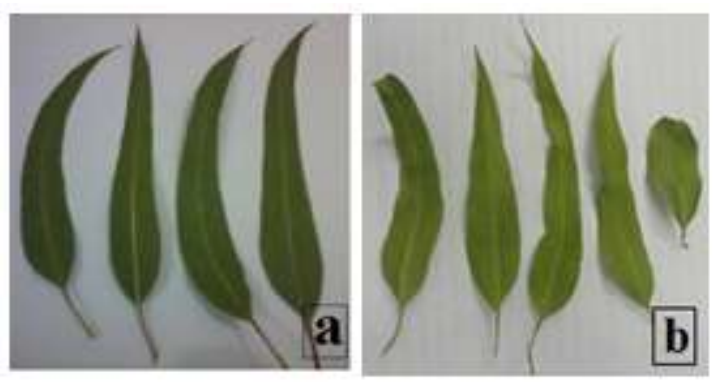

Figura 1 - Folhas in natura e secas.

Extração utilizando Soxhlet: Para esta metodologia foi utilizado o extrator apresentado na Figura 2. Como solventes foram utilizados hexano e água. Foram pesados aproximadamente $10 \mathrm{~g}$ de amostra (in natura e seca) e envolvidas em papel filtro, formando uma "cápsula", que foi depositada no tubo extrator. Introduziu-se $200 \mathrm{~mL}$ do solvente no balão e, logo após, ajustou-se a temperatura da chapa de aquecimento. O experimento teve duração de $2 \mathrm{~h}$, após o ponto de ebulição de cada solvente. Após o término do procedimento, retirou-se o balão do aparato e adicionou-se $50 \mathrm{~mL}$ de diclorometano no experimento realizado com água, que tem por finalidade a separação da fase aquosa e da fase oleosa. Em seguida, a amostra foi levada ao rotaevaporador (Figura 3), cuja chapa é ajustada à temperatura de evaporação do solvente utilizado. Ao término desta etapa, coloca-se a amostra em estufa, por $30 \mathrm{~min}$, com o objetivo de retirar completamente o residual de solvente. Todas as análises foram feitas em duplicatas.

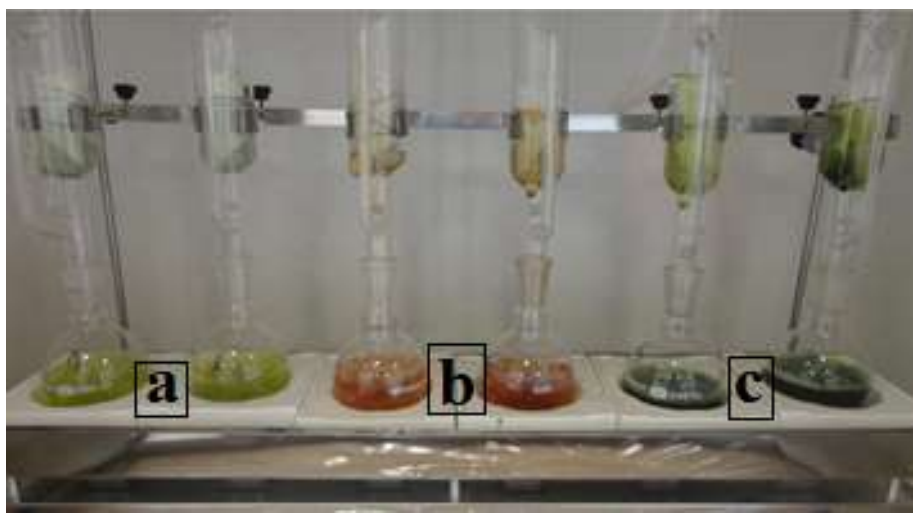

Figura 2 - Extrator Soxhlet. 


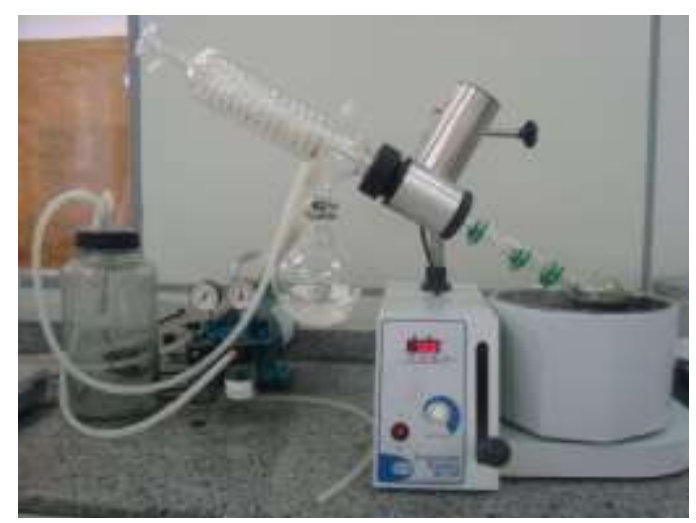

Figura 3 - Rotaevaporador utilizado.

Extração por infusão: A extração por infusão foi realizada utilizando o aparato de destilação simples apresentado na Figura 4, e como solvente água. Inicialmente pesou-se $10 \mathrm{~g}$ de amostra de folhas de eucalipto em um balão e adicionou-se $400 \mathrm{~mL}$ de água destilada. $\mathrm{O}$ experimento teve duração de 2 h, a partir do instante em que atingiu-se o ponto de ebulição da água. Após a extração adicionou-se $50 \mathrm{~mL}$ de diclorometano para a separação entre a fase aquosa e oleosa. A mistura foi então transferida para um funil de separação e separadas as fases Após retirada da água, utilizou-se o rotaevaporador para separar o óleo e o diclorometano. Após isto, colocou-se o balão na estufa, para a retirada de todo residual do solvente.

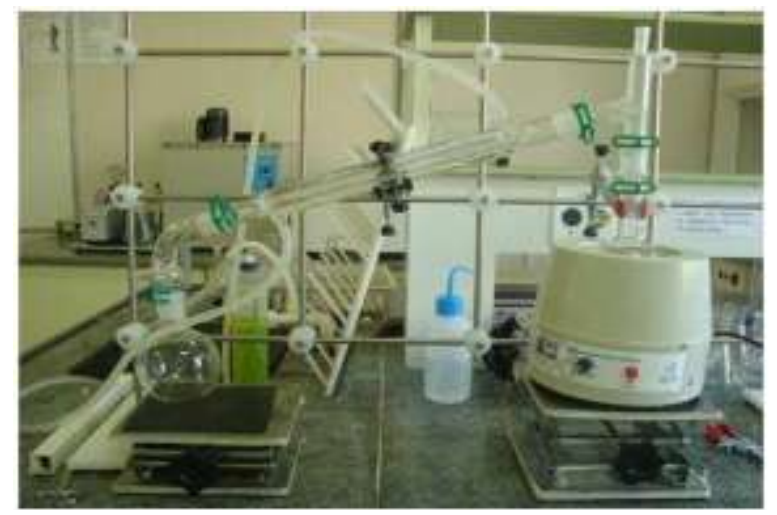

Figura 4 - Destilador simples.

Determinação dos fenóis totais: A quantificação dos compostos fenólicos foi determinada segundo método Singleton e Rossi (1965), com algumas modificações. Inicialmente pesou-se $1 \mathrm{~g}$ de amostra triturada, diluindo-se em $50 \mathrm{~mL}$ de metanol, seguido da homogeneizado por $30 \mathrm{~min}$ em mesa agitadora, prosseguindo da filtração à vácuo. Para caracterização dos fenóis totais foi retirada uma alíquota $1 \mathrm{~mL}$ do extrato obtido e adicionado $10 \mathrm{~mL}$ de água destilada e $1 \mathrm{~mL}$ de reagente de Folin-Ciocalteau, deixou-se reagir por $3 \mathrm{~min}$, após adicionou-se uma solução de carbonato de cálcio 3,75\%, deixando assim repousar por mais $2 \mathrm{~h}$ no escuro. Efetuou-se a leitura da absorbância em espectrofotômetro com comprimento de $760 \mathrm{~nm}$, utilizando como branco água destilada. A Figura 5 apresenta equipamento utilizado para caracterização dos fenóis, a análise foi realizada em triplicata e os resultados expressos em $\mathrm{mg}$ de ácido gálico.g $\mathrm{g}^{-1}$ de amostra. 


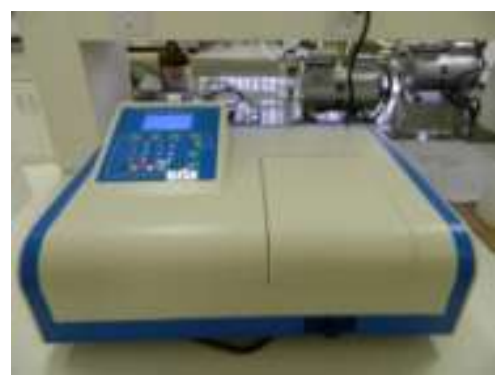

Figura 5 - Espectrofotômetro (UV 755B).

Determinação das clorofilas: Para determinação da clorofila foi realizado o método citado por Lichtenthaler (1987), com algumas modificações. Pesou-se aproximadamente $1 \mathrm{~g}$ de amostra triturada, acrescentando-se $10 \mathrm{~mL}$ de uma solução de $80 \%$ acetona (v/v). Após, centrifugou-se em $4000 \mathrm{~g}$ por $10 \mathrm{~min}$ e o sobrenadante foi após transferido para um balão volumétrico de 25 $\mathrm{mL}$, completando o volume com a solução de acetona a $80 \%$ (v/v). Realizou-se a leituras em espectrofotômetro com os comprimentos de onda a $647 \mathrm{~nm}$ e $663 \mathrm{~nm}$, utilizando como branco acetona $80 \%(\mathrm{v} / \mathrm{v})$. Os resultados foram expressos em mg. $100 \mathrm{~g}^{-1}$. Utilizando as Equações 1 e 2, calcularam-se os conteúdos de clorofila $a$ e $b$, e através da Equação 3, determinou-se o conteúdo de clorofilas totais.

$$
\begin{aligned}
& \text { Clorofila } a=12,25(A 663)-2,79(A 647) \\
& \text { Clorofila } b=21,50(A 647)-5,10(A 663) \\
& \text { Clorofilas Totais }=7,15(A 663)+18,71(\text { A647) }
\end{aligned}
$$

em que $\left(\mathrm{A}_{663}\right)$ e $\left(\mathrm{A}_{647}\right)$ são as absorbâncias nos respectivos comprimentos de onda.

\section{RESULTADOS E DISCUSSÃO}

Os resultados para o rendimento de óleo essencial da espécie Eucalyptus citriodora foram entre 0,09 e 7,9 \% (b.u.). A Tabela 1 apresenta os valores obtidos para o rendimento com diferentes técnicas de extração e solventes, para as amostras in natura e seca. 
Tabela 1 - Rendimento do óleo essencial das folhas de eucalipto

\begin{tabular}{|c|c|c|c|c|}
\hline \multirow{2}{*}{ in natura } & b.u. & Infusão água & Soxhlet hexano & Soxhlet água \\
\cline { 2 - 5 } & b.s. & $4,80 \%$ & $3,16 \%$ & $0,17 \%$ \\
\hline \multirow{2}{*}{ Seca } & b.u. & $3,95 \%$ & $7,74 \%$ & $0,43 \%$ \\
\cline { 2 - 5 } & b.s. & $4,16 \%$ & $7,90 \%$ & $0,09 \%$ \\
\hline
\end{tabular}

Através da Tabela 1 observa-se que os resultados da extração com Soxhlet utilizando água foram menores que os obtidos pelo método de infusão com água, enquanto que o uso do Soxhlet com hexano permitiu extrair mais óleo. Segundo Kier (1996), que estudava o melhoramento dos níveis de rendimento do Eucalyptus citriodora, a extração do óleo essencial das folhas in natura utilizando a infusão é em torno de $1 \%$ (b.u.), o que condiz com o resultado obtido neste trabalho. De acordo com a literatura o óleo essencial desta espécie de eucalipto tem como componente principal a citronela, com conteúdo na faixa de 65 a $85 \%$ (Ciniglio, 1993).

Os resultados referentes ao rendimento obtido através da extração em Soxhlet encontram-se superiores ao reportado por Mochi (2005), em que o rendimento do óleo essencial para a folha de eucalipto in natura foi $0,23 \%$ (b.u.), e para as folhas secas foi de $1,22 \%$ (b.u.), utilizando o extrator Soxhlet e tendo como solvente o hexano. Entretanto, os resultados apresentam valores semelhantes ao reportado por Ricardo e Rosa (2012), em que utilizando o mesmo tipo de extrator e solvente as folhas in natura e secas a $60{ }^{\circ} \mathrm{C}$ apresentaram rendimento de óleo de 3,7 e 7,6 \% (b.u.), respectivamente. $\mathrm{O}$ valor obtido no presente trabalho, em base seca, para a extração utilizando Soxhlet com hexano em amostras secas foi maior que para a amostra in natura, o que pode estar associado a facilidade de extração do óleo sem a presença majoritária da água na estrutura da matéria-prima (Braga et al., 2004)

As Tabelas 2 e 3 apresentam os resultados das análises de fenóis totais e clorofilas presentes nas folhas de eucalipto.

Tabela 2 - Fenóis totais para folhas in natura e secas

\begin{tabular}{|c|c|c|}
\hline \multirow{2}{*}{ in natura } & & mg. $\mathrm{g}^{-1}$ \\
\cline { 2 - 3 } Seca & b.u. & $8,88 \pm 0,04$ \\
\hline \multirow{2}{*}{ b.s } & b.u. & $19,66 \pm 0,10$ \\
& b.s. & $9,64 \pm 0,08$ \\
\hline
\end{tabular}

Valores médios \pm desvio médio $(\mathrm{n}=3)$ 
Tabela 3 - Análise do conteúdo de clorofila $\left(\mathrm{mg} .100 \mathrm{~g}^{-1}\right)$ para folhas in natura e secas

\begin{tabular}{|c|c|c|c|}
\hline & Clorofila "a" & Clorofila "b" & Clorofilas totais \\
\hline in natura & $25,97 \pm 0,009$ & $9,57 \pm 0,340$ & $35,56 \pm 0,347$ \\
\hline Seca & $21,13 \pm 0,241$ & $7,12 \pm 0,469$ & $28,26 \pm 0,288$ \\
\hline
\end{tabular}

Valores médios \pm desvio médio $(n=3)$

Pode-se observar que o conteúdo de fenóis totais diminuiu para as amostras secas (b.s.), quando comparado a amostra in natura, sendo observado a perda de $46 \%$ destes. Para o conteúdo de clorofila também observaram-se decréscimos. Este comportamento pode estar relacionado ao processo de secagem em estufa com temperatura de $60{ }^{\circ} \mathrm{C}$, que pode ter degradado parte dos componentes bioativos. De acordo com Caneda (2013), os compostos bioativos de uma forma geral, são caracterizados por serem termossensíveis, e portanto a temperatura de processamento é um fator importante a se considerar na retenção destes compostos.

Segundo Vieira (2013), os valores médios de clorofilas "a", "b" e totais para folhas de Eucalyptus citriodora foram de 26,03, 8,81 para clorofila "a" e "b", respectivamente, e 34,84 mg. $100 \mathrm{~g}^{-1}$ para o conteúdo de clorofilas totais. Os valores encontrados no presente trabalho, como observado na Tabela 3, encontram-se próximos dos valores reportados.

\section{CONCLUSÃO}

Foi verificado que a extração do óleo essencial apresentou maior rendimento após as folhas de eucalipto passarem pelo processo de secagem. Analisando os resultados verificamos que os rendimentos obtidos foram maiores do que consta na literatura. $\mathrm{O}$ método de extração do óleo essencial que se mostrou mais eficiente foi através do uso do extrador Soxhlet, usando hexano como solvente. Os valores para o conteúdo de fenóis totais foram entre 8,9 e 9,6 mg.g ${ }^{-1}$ (b.u.) e de clorofila totais foram entre 28,3 e 35,6 mg. $100 \mathrm{~g}^{-1}$.

\section{REFERÊNCIAS BIBLIOGRÁFICAS}

BRAGA, N. P; BRITO, J. O.; CREMASCO, M. A. Estudo da secagem das folhas de Eucalyptus Staigeriana por meio do acompanhamento da umidade do ar de descarga do secador. Anais do XV COBEQ, Congresso Brasileiro de Engenharia Química, Curitiba, 2004.

BRITO, J. O. Goma-resina de pinus e óleos essenciais de eucalipto: Destaques na área de produtos florestais não-madeireiros. Tecnologia de produtos florestais, LCF/ESALQ/USP, Brasil, set., 2002.

CANEDA, C. M. Secagem da pimenta vermelha dedo-de-moça (Capsicum baccatum var. Pendulum): compostos bioativos e propriedades antioxidantes, Trabalho de Conclusão de 
Curso-EQ, Unipampa, Bagé, 2013.

CINIGLIO, G. Eucaliptos para produção de óleos essências, ESALQ-USP, Departamento de Ciências Florestais, Piracicaba, 1993.

CORRÊA, R. M.; BERTOLUCCI, S. K. V.; PINTO, J. E. B. P.; REIS, E. S.; ALVES, T. L. Rendimento de óleo essencial e caracterização organoléptica de folhas de assa-peixe submetidas a diferentes métodos de secagem. Ciênc. Agrotec., Lavras, v. 28, p.341346.,2004.

KIER, H. Exploração de plantas aromáticas e óleos essenciais, Simpósio de óleos essências I, SP. Fundação Cargill, p.15-20, 1986.

LICHTENTHALER, H. K. Chlorophylls and carotenoids: pigments of photosynthetic biomembranes. Methods Enzymol., v. 148, p. 350-381, 1987.

MOCHI, V. T. Efeito da temperatura de secagem no rendimento do óleo essencial e teor de 1,8 cineol presente nas folhas de Eucalyptus camaldulensis, Dissertação de mestrado EQ, Unicamp, Campinas, 2005.

RICARDO, L.; ROSA, G. S da. Influência da temperatura do ar de secagem no rendimento do óleo essencial da folha de eucalipto Anais do XXIV SIC, Salão de Iniciação Científica da UFRGS, Porto Alegre, 2012.

ROCHA, M. E. N; SANTOS, C. L.; Universidade do Grande Rio - Unigranrio, Saúde \& Ambiente em Revista, Duque de Caxias, v.2, n.2, p.23-24, jul/dez, 2007.

SINGLETON, V. L.; ROSSI, J. A. Jr. Colorimetry of total phenolics with phosphomolybdicphosphotungstic acid reagents. Amer. J. Enol. Viticult., vol. 16, p. 144-158, 1965.

VIEIRA, T. O. Plasticidade Fenotípica e Aclimatação de Siparuna guianensis em respostas a gradiente de luz. Dissertação de mestrado em Ecologia e Recursos Naturais, UENF, Campos dos Goytacazes, agosto, 2013.

\section{AGRADECIMENTOS}

A Universidade Federal do Pampa pelo apoio e a Fundação de Amparo à Pesquisa do Estado do Rio Grande do Sul (FAPERGS) pela bolsa de iniciação científica. 